\title{
Gestión y Liderazgos en los Emprendimientos Sociales: El caso del Sector No Lucrativo Chileno $^{1}$
}

\author{
Mario H. Radrigán R.
}

Centro Internacional de Economía Social y Cooperativa, Facultad de Administración y Economía, Universidad de Santiago, Chile. Email: mario.radrigan@usach.cl

\section{Ana María Dávila R.}

Centro Internacional de Economía Social y Cooperativa, Facultad de Administración y Economía, Universidad de Santiago, Chile. Email: anamaria.davila@usach.cl

\section{Francesco E. Penaglia V.}

Centro Internacional de Economía Social y Cooperativa, Facultad de Administración y Economía, Universidad de Santiago, Chile. Email: francesco.penaglia@usach.cl

Resumen: Las organizaciones sin fines de lucro han sufrido grandes cambios. Dejó de ser un sector empobrecido, donde se recibían como donaciones aquel dinero que les sobraba a los grandes empresarios, y donde sólo tenían cabida las personas voluntarias. Hoy en día es un sector marcado por el trabajo de personas profesionales y por ser organizaciones capaces de llenar los espacios que ha dejado el Estado sin atender. Además de ser organizaciones inteligentes, con estructuras flexibles, que les permiten responder de manera ágil a los requerimientos de su entorno. Sin embargo para que el sector sin fines de lucro pueda evolucionar de manera exitosa, no sólo necesitan de la labor de sus miembros, también necesitan del compromiso del estado, las universidades y las entidades privadas. Un compromiso que se haga visible a través de alianzas de colaboración entre sí, y así de esta manera trabajar conjuntamente en el desarrollo humano y la inclusión social en Chile.

Palabras clave: Economía Social, emprendimientos sociales, organizaciones no lucrativas, gestión y liderazgo.

\section{Management and Leadership in Social Entrepreneurship: The case of Chilean Nonprofit Sector}

Abstract: The not-for-profit organizations have been suffering great changes. I stopped being impoverish sector, receiving the leftover money from the big businessman, and where only volunteer's could participate. Nowadays it is a sector characterized for hiring professionals and capable of filling those spaces the State, has left without attention. Besides being clever organizations with flexible structures that allow them to respond speedily to the environment requirements. 
However, for this sector to evolve successfully, they need not only the labor of their members, but also the compromise of the State, universities and private entities. A commitment made visible through collaboration alliances, and through that work together in order to achieve social inclusion and human development in Chile.

Key words: social economy, social enterprises, nonprofit organizations, management and leadership.

\section{Gestão e Liderança em Empreendedorismo Social: O caso do Terceiro Setor chileno}

Resumo: As organizações sem fins lucrativos têm sofrido grandes mudanças. Eles não estão focados exclusivamente no setor empobrecido, quando foi utilizado somente para receber o dinheiro remanescente do grande empresário, e que só tinha espaço para os voluntários. Hoje é um setor marcado pelo trabalho de profissionais e organizações para ser capaz de preencher os espaços deixados sem atenção pelo Estado. Além de ser organizações de aprendizagem, estruturas flexíveis que lhes permitam responder rapidamente às exigências do seu ambiente. No entanto, para a indústria sem fins lucrativos podem evoluir com sucesso, não só precisa do trabalho dos seus membros, também precisa do compromisso do Estado, universidades e entidades privadas. Um compromisso torna-se visível através de parcerias entre si, e assim desta forma a trabalhar juntos no desenvolvimento humano e inclusão social no Chile.

Palavras-chave: economia social, empresas sociais, organizações sem fins lucrativos, gestão e liderança.

\section{Introducción}

La Economía Social (ES) es un subsector de la economía, que posee ciertas características particulares, entre estas la más significativa está relacionada con la primacía del hombre y del objeto social por sobre el capital. Característica que la diferencia del sector público y de las organizaciones tradicionales. Otras denominaciones que recibe la Economía Social son: Tercer Sector, Economía Solidaria, Economía Participativa, Sector No Lucrativo, etc.

Dentro de la Economía Social se encuentran las organizaciones no lucrativas, las que cumplen como objetivo principal producir servicios nos destinados a la venta, satisfaciendo la necesidad de sus asociados en diversos ámbitos como el desarrollo rural, cultura, educación, salud, adulto mayor, infancia, entre otros.

En una mirada amplia, es posible señalar que el sector no lucrativo chileno se encuentra fuertemente desarrollado y ha sufrido un importante crecimiento durante los últimos 30 años, en el contexto de un Estado subsidiario y una sociedad civil que se organiza para llenar los espacios dejados por el mercado. Asimismo, el estudio realizado por la Universidad de Johns Hopkings evidencia que Chile posee el mayor sector sin fines de lucro de 
Latinoamérica, duplicando el tamaño de Brasil y Colombia. A su vez el informe, caracterizó al sector no lucrativo como un actor central en la economía, empleando en forma remunerada y voluntaria a 303 mil personas en jornada completa, representando el 2,6\% del empleo remunerado y aportando al 1,5\% del PIB (PNUD-FOCUS; 2006).

Sin embargo, pese a la importancia social y económica, el sector no lucrativo ha sido muy poco estudiado, encontrándose una escasa integración de datos y catastro de las organizaciones; a la vez que se observa una baja investigación cualitativa e identificación de los fenómenos organizacionales, sobre todo en materias de gestión, liderazgo e innovación. Es por ello que el objetivo central de este documento es contribuir al desarrollo teórico de estas materias, presentando los resultados preliminares de una investigación realizada por CIESCOOP (Centro Internacional de Economía Social y Cooperativa) con apoyo del Departamento de Investigación Científica y Tecnológica, DICYT, de la Universidad de Santiago de Chile (USACH), denominada "Emprendimientos sociales y liderazgo en el sector no lucrativo chileno. Modelos de gestión y buenas prácticas”.

\section{Revisión Bibliográfica}

\section{Economía Social y Sector No Lucrativo}

Las entidades de la Economía Social, son aquellas organizaciones que (por lo habitual) tienen una gestión democrática y desarrollan sus actividades orientadas al interés general y de sus asociados o beneficiarios; a su vez tienen una fuerte presencia tanto en los países de América Latina como de la Unión Europea, con formas jurídicas que corresponden por lo general a mutuales, fundaciones, cooperativas y asociaciones comunitarias de diverso tipo, desarrollando actividades en amplios sectores de la vida económico y social.

La Comisión Científica del CIRIEC-España propone definir la Economía Social como el "conjunto de empresas privadas creadas para satisfacer las necesidades de sus socios a través del mercado, produciendo bienes y servicios, asegurando o financiando y en las que la distribución del beneficio y la toma de decisiones no están ligadas directamente con el capital aportado por cada socio, correspondiendo un voto a cada uno de ellos. La Economía Social también incluye a las instituciones sin fines de lucro que son productores no de mercado privado, no controlados por las Administraciones Públicas y que producen servicios no destinados a la venta para determinados grupos de hogares, procediendo sus recursos principalmente de contribuciones voluntarias efectuadas por los hogares en su calidad de consumidores, de pagos de las Administraciones Públicas y de rentas de la propiedad” (Monzón, 2004, p.18).

Al interior de la Economía Social es posible distinguir entre dos subsectores: de mercado, con distribución democrática de los beneficios 
(ej. Cooperativas); y un subsector de no mercado o no lucrativo, al servicio de los hogares (ej. Organizaciones comunitarias).

Las organizaciones del sector social, son organizaciones que no cumplen con los atributos básicos para incluirse ni en la economía pública, ni tampoco entre las entidades tradicionales, tienen entre sus características fundamentales: a) la primacía del hombre y del objeto social sobre el capital; b) la adhesión voluntaria y abierta, y el control democrático de sus miembros desde la base; c) la conjunción de los intereses de los miembros y del interés general; d) la defensa y aplicación del principio de solidaridad y responsabilidad; e) la autonomía en la gestión y la independencia de los poderes públicos; f) la creación de empleo estable y de de calidad; g) la aproximación de los trabajadores al núcleo de decisión de la empresa; y h) la formación de una conciencia de autorresponsabilidad de los propios trabajadores (Barría y Radrigán, 2005).

Según el informe "La Economía Social en la Unión Europea” elaborado en el marco del Consejo Económico y Social Europeo el año 2008, las entidades de la Economía Social generan más de 11 millones de puestos de trabajo y sus asociados tienen un peso significativo a nivel de la sociedad y la economía. En América Latina no existen estadísticas tan certeras, pero un estudio realizado por FUNDIBES el año 2009 arroja provisionalmente la existencia de más de 700.000 organizaciones de la Economía Social, con cerca de 14 millones de asociados, y que tienen sus raíces en procesos histórico-sociales por más de un siglo y medio, lo que nos permite aseverar que tienen una importante implantación y raigambre en las distintos países de Europa y América Latina.

En Chile no existe a la fecha una cuantificación definitiva de las organizaciones de la economía social y solidaria. De esta manera, las aproximaciones con que se cuenta provienen de dos grandes fuentes. De una parte los datos entregados por el Informe de Desarrollo Humano 2000 donde se elabora un "mapa de asociatividad" que registró 83.386 organizaciones de sello asociativo (PNUD Chile, 2000, p.303). Por otra parte se sitúan los datos del estudio comparativo para el sector sin fines de lucro, que contabilizó 106.880 organizaciones clasificadas de acuerdo al código de actividad ICPNO (International Classification of Nonprofit Organizations) (PNUDFOCUS; 2004).

El sector no lucrativo, según Drucker (2001) constituyen un elemento medular de la sociedad, existiendo para generar un cambio en los individuos y la sociedad. Asimismo asegura que, la institución sin fines de lucro no se limita a prestar un servicio, buscando que el usuario final no sea un consumidor, sino un participante activo, capaz de producir un cambio social. El autor agrega también que, casi por definición estas organizaciones siempre andan escasas de fondos, por ello el propósito de una estrategia de recaudación de fondos es precisamente posibilitar el cumplimiento de la misión sin subordinarla. 
De igual forma, el enfoque del Sistema Europeo de cuentas económicas 1995, define a las instituciones sin fines de lucro como "entidades legales o sociales creadas con el fin de producir bienes y servicios y cuya condición no le permite ser fuentes de rentas, beneficios u otras ganancias financieras por las unidades que la establecen, controlan o financian. En la práctica, las actividades productivas de las OSFL generan excedentes o pérdidas, pero las otras unidades institucionales no pueden apropiarse de los posibles excedentes” (Barea y Pulido, 2011).

Coleman (1990; citado en: Universidad de Johns Hopkins, 2006) asegura que, las instituciones sin fines de lucro son creadas con la contribución del "capital social”, entendido como un vínculo de confianza y reciprocidad que resulta crucial para que una democracia y una economía de mercado funcionen de manera efectiva. El capital social, al establecer conexiones entre individuos y al participar en asociaciones, enseña normas de cooperación que influyen en la vida política y económica.

Según la literatura anglosajona las entidades no lucrativas se caracterizan por cinco requisitos básicos propuestos por Salomon y Anheier (1992) (citado en: Barea y Pulido, 2001): a). Organización formal, la entidad debe estar estructurada con objetivos claramente establecidos, identificación de su masa social y con un estatuto legal específico; b). Privada, institucionalmente separada del Sector Público, sin que este pueda nombrar a sus administradores ni establecer su política general, aunque pueda tener apoyo financiero público; c). No lucrativa, lo que significa que no puede distribuir beneficios a las personas que controlan la entidad. Si se obtienen beneficios se destinan al desarrollo de sus fines o se reinvierten; d). Autogobierno de la propia entidad, disponiendo ésta de una regulación propia de sus órganos de gobierno que no pueden estar sometidos a otras entidades, ni públicas ni privadas; y e). Participación voluntaria en el desarrollo de sus actividades, lo que supone que la entidad utiliza trabajo no remunerado, o donaciones altruistas sin perjuicio de que también se contrata en el mercado factores productivos remunerados.

Autoras como Lilia Barrionuevo (2001), consideran que, las ONG vienen a cubrir, muchas falencias no deseadas del desarrollo y la globalización. La teoría "del derrame” (el progreso se derramaría hacia los más pobres) no ha llegado a tener el éxito previsto por los economistas neoliberales. Ello implica un cambio rotundo, entendiendo que el mercado no puede resolver el tremendo problema de la pobreza y de la exclusión. Las ONG insertas en el mundo, han superado los conceptos de voluntariado y beneficencia y en muchos casos han reemplazado con eficiencia al propio estado. Pero ya no son pequeñas organizaciones de voluntarios, creciendo marcadamente en número (aprox. 78,4 mil en Argentina - PNUD y BID, Setiembre'98) y siendo considerados actores claves de la nueva economía. El sector no lucrativo es llamado a ser el sector más trascendente del siglo XXI y sus influencias se extiende tanto a los países desarrollados, como en vías de desarrollo (en EEUU, en las últimas décadas del siglo XX, ya representaban el 7,7\% del ingreso nacional y comprendían el 10,9\% del empleo total - Wolch, 1987). 


\section{Emprendimientos Sociales}

Según un estudio realizado por el Banco Interamericano de Desarrollo y Social Enterprise Knowledge Network; "Gestión efectiva de emprendimientos sociales. Lecciones extraídas de empresas y organizaciones de la sociedad civil en Iberoamérica” (BID y SEKN, 2006), el concepto de emprendimiento social se relaciona directamente con la acción de contribuir a acelerar el proceso de mejora de la humanidad. Asimismo, cuando las compañías llevan a cabo acciones tendientes exclusivamente a la creación de valor social, ingresan en el ámbito de las empresas sociales. En la realización de un emprendimiento social, la empresa tiene la intención explícita de generar mejoras sociales a través de la movilización y asignación inteligente de sus recursos. De hecho, las mejoras sociales afectan el contexto donde operan las compañías, que se benefician directamente gracias a las iniciativas sociales que ayudan a desarrollar. El estudio sugiere que muchas veces los emprendimientos sociales se desarrollan en cuatro etapas, comenzando como iniciativa de un individuo, quien al identificar un problema de orden social, económico o ambiental, desarrolla un proyecto para contribuir a su solución.

Siguiendo por la misma línea del estudio, es importante resaltar que se habla de dos habilidades necesarias, que deben tener en un inicio las personas que desean realizar un emprendimiento social; como primera medida se hace mención de la habilidad emprendedora, la cual se define como la capacidad de un individuo para identificar y aprovechar oportunidades que le permitan iniciar un emprendimiento, independientemente de los recursos que tenga bajo su control. Y en segundo lugar encontramos la habilidad de diagnóstico, la que se define como la capacidad para estudiar con rigor y comprender el conjunto de causas y consecuencias, en el corto, mediano y largo plazo, de los problemas sociales que aquejan el entorno).

Después de analizar las condiciones de su entorno, frecuentemente el líder diseña un plan para dar respuesta a un problema, lo que resulta determinante en la fundación del emprendimiento. La habilidad de diagnóstico comprende la articulación de una solución factible y efectiva, a través de un modelo de cambio social. El diagnóstico social puede servir para aunar voluntades y generar consenso. A partir de esta habilidad los líderes desarrollan una teoría de cambio a partir de la cual intentan modificar la realidad. Una teoría de cambio consiste en una proposición convincente a cerca de cómo el uso de insumos en un emprendimiento generará resultados a largo y corto plazo, especificando la cascada de causas y consecuencias que producirán el efecto deseado (BID y SEKN, 2006).

\section{Metodología}

Este artículo se crea en el marco del proyecto DICYT (Departamento de Investigación de Ciencia y Tecnología de la Universidad de Santiago de Chile) 2010 - 2012 "Emprendimientos sociales y liderazgo en el sector no lucrativo chileno. Modelo de gestión y buenas prácticas"; que se en- 
cuentra actualmente en ejecución. El estudio que se ha realizado es de carácter cualitativo y ha utilizado como herramientas de recolección de la información la revisión bibliográfica y los casos de estudio a través de técnicas documentales, entrevistas a profundidad con informantes claves y observación en terreno.

Para la realización de este artículo se contó con el estudio de 17 casos: 6 fundaciones, 3 corporaciones y 8 organizaciones comunitarias. El levantamiento de los casos se realizó desde Noviembre de 2010 hasta septiembre de 2011.

El método utilizado en este estudio es la investigación exploratoria, pues la muestra con la que se cuenta actualmente no es significativa, lo que permite solo tener una visión aproximada de la realidad de estas organizaciones, es por esta razón que lo que se busca en este artículo es generar una discusión de resultados, con los hallazgos encontrados en los estudios de caso respecto a los temas de gestión y liderazgo y con los datos secundarios aportados por la bibliografía utilizada en este artículo.

Para poder realizar el análisis de la información y la discusión de resultados, se dividieron los hallazgos en dos ejes: 1). Gestión, que contiene las temáticas de: recursos humanos; adaptación al medio e innovación; vínculos, colaboración y financiamiento; sentido de pertenencia, y comunicación. 2). Liderazgo, que contiene las temáticas de: planificación; y liderazgo y toma de decisiones.

\section{Discusión de resultados}

\section{Recursos Humanos y Gestión del Conocimiento}

En cuanto al tema relacionado con la profesionalización de las personas que trabajan en estas organizaciones, las entrevistas en general demostraron que, se presenta un alto nivel de profesionalización tanto en las labores relacionadas con la gestión y la administración; como también las actividades de campo. Gran parte de las personas que trabajan en estas organizaciones son profesionales del área de las Ciencias Sociales y Humanidades.

Por ejemplo en el caso de la Fundación Asciende, afirma que, "las personas que son parte de la organización son profesionales de las ciencias sociales y humanidades, la mayoría han realizado estudios en: sociología, antropología, trabajo social, periodismo y docencia, entre otros”.

Otro ejemplo claro es el del canal comunitario Pochilemu TV, que expone "uno de los pilares fundamentales de Pichilemu TV que lo diferencia de las antenas populares, es que sus creadores parten de una base universitaria, por lo que poseen conocimientos audiovisuales, haciendo que el trabajo sea más profesional. A pesar de que los voluntarios son totalmente 
autodidactas, los creadores ayudan y enriquecen los programas cuando consideran que tienen falencias”.

En el estudio realizado por Kliksberg (2011), se asegura que el tema formativo es decisivo para el futuro de los emprendimientos sociales, asimismo afirma que, proporcionar en forma sistemática, a través de instituciones especializadas, y del mundo de las universidades, una formación sólida, es uno de los desafíos centrales para su avance. Por otro lado, Paul Light (2008) (citado en: Kliksberg, 2011), quien investigó 131 organizaciones sociales de alto desempeño, encontró que su éxito dependía más que de la personalidad de sus fundadores, de la disciplinada aplicación de habilidades y conocimientos de liderazgo, y emprendimiento enseñables.

Respecto al tema del voluntariado, Barrionuevo (2001), afirma que la cooperación no es una labor de aficionados, porque la calidad, los riesgos y la magnitud de las necesidades que se afrontan, exigen que las personas que trabajen en este campo estén preparadas y sean verdaderos profesionales. La experiencia demuestra que un trabajo llevado a cabo exclusivamente por voluntarios, resta eficiencia a la labor. Los que trabajan por la satisfacción de las necesidades comunitarias no son héroes o masoquistas, son profesionales que tratan de hacer su trabajo de la mejor manera posible.

Un ejemplo de lo anterior es lo expuesto por la Fundación Paternitas, quienes aseguran que, "la organización trabaja con profesionales universitarios, si bien en un comienzo la organización nutrió su actuar con voluntariado, esto se desechó rápidamente, para poder realizar un trabajo serio y responsable con profesionales que se dedicaran con exclusividad a las labores de la organización”.

Otro ejemplo importante de resaltar es el de la Fundación Trascender, quienes afirman que "la organización apunta a una profesionalización del voluntariado, por lo tanto trabajan con personas tituladas, generalmente en un rango de 30 a 40 años, aunque también existe un grupo de voluntarios jubilados, que se ha ido incrementando con el tiempo. El factor central para la organización es la experiencia, lo que permite enviar a ciertos voluntarios a organizaciones y proyectos acordes con los conocimientos prácticos que los participantes poseen”.

Sumado a lo anterior está claro que el rol del voluntariado en las organizaciones sin fines de lucros es importante, pues según el estudio realizado por la Universidad de Johns Hopkins (2006), en Chile, un promedio de 7\% de la población adulta aportó durante el 2004 parte de su tiempo al trabajo voluntario. Cada trabajador voluntario destinó, en promedio, un cuarto de jornada al sector sin fines de lucro. Esto se traduce en $143 \mathrm{mil}$ trabajos de tiempo completo adicionales, equivalentes al 2,3\% de la población económicamente activa, lo que es un significativo aporte al desarrollo y permanencia de la sociedad civil chilena. De igual forma el empleo total de la sociedad civil está compuesto en un $47 \%$ por trabajadores volunta- 
rios, con lo que Chile es lejos el país que presenta mayor participación del voluntariado en Latinoamérica y se encuentra incluso por sobre el nivel de los países desarrollados.

Otro hallazgo importante dentro del eje de recursos humanos, es el tema de la gestión del conocimiento, considerado por estas organizaciones como un objetivo estratégico, y en el caso particular de las organizaciones comunitarias (uniones comunales, preuniversitarios populares, canales comunitarios entre otros) la herramienta del autoaprendizaje ha jugado un rol muy importante debido a que no todos los miembros que conforman las distintas experiencias son profesionales, sin embargo han creado capacidades e implementado estrategias para dotarse con los conocimientos necesarios para desempeñar las funciones fundamentales.

Algunos ejemplos que confirman lo anterior son: Fundación Paternitas, "la gestión del conocimiento es considerado como un objetivo estratégico al que hay que llegar a través de la experiencia que se genera con el trabajo diario”.

Taller de Acción Comunitaria Valparaíso, "la gestión del conocimiento explica como las organizaciones deben incorporar nuevas formas de aprendizaje y recoger las ya existentes para subsistir en un mundo de alta competencia y expuestos a constantes cambios, transformando así su conocimiento en un recurso disponible. El conocimiento que se genera al interior de la organización se traspasa, ya que el TAC (Taller de Acción Comunitaria) se define como una experiencia compartida de aprendizaje”.

Fundación Víctor Jara, "La gestión del conocimiento se basa en la teoría de pensar que las organizaciones se encuentran compuestas por personas que poseen conocimientos, y que estos conocimientos deben ser identificados, organizados y transmitidos en la organización, de forma permanente en el tiempo. Este aprender a aprender en las organizaciones se institucionaliza y regulariza como un proceso, mientras que en las organizaciones no tradicionales como es el caso de la Fundación Víctor Jara, no se concibe como un proceso administrativo, no se generan instancias donde se discuta sobre la gestión del conocimiento, si no, más bien, al igual que en la gestión del talento humano, se da de forma natural, no en su totalidad, pero en rasgos generales, al ser una organización donde prevalecen los valores democráticos, abiertos al dialogo, las posibilidades de identificar las buenas ideas o de mejorar la forma de hacer las labores, se dan a través del constante dialogo y participación de los integrantes de la fundación. La creación de nuevo conocimiento es siempre una posibilidad, la innovación, el compartir los aprendizajes, se lleva a cabo, por las relaciones participativas que se dan dentro de la organización como con el entorno con el que trabajan”.

Para Barrionuevo (2001) el conocimiento es el nudo principal y verdaderamente estratégico de estas organizaciones, tiene un papel importantísimo en el crecimiento económico y social. Se define al creci- 
miento como al desarrollo del conocimiento, llevado a cabo por quienes tienen a su cargo las tareas productivas o de generación de valor. Las instituciones exitosas destinan una porción importante de sus recursos a la capacitación, profesionalizando su operación y administración; y desarrollan procedimientos para nutrirse de la inteligencia del medio en el que actúan, capitalizándola para generar valor. Les compete a estas organizaciones, la misión de trasvasar al proceso productivo, las externalidades positivas que genera la investigación y el desarrollo.

Por otro lado, el estudio realizado por el BID y SEKN (2006), expone que, el aprendizaje organizacional está directamente relacionado con los siguientes puntos: 1) comunicación y coordinación; 2) documentación memoria institucional; 3) Mecanismos de evaluación del desempeño; 4) compartir información; 5) sistema de mentores; y 6) liderazgo y aprendizaje.

\section{Gestión adaptación al medio e innovación:}

Al hablar de la adaptación al medio, indudablemente es necesario hablar del cambio, pues esta claro que no existe adaptación sin la generación de un cambio o innovación. Asimismo el modo en que los cambios del entorno han exigido la modificación o el replanteo de las estrategias organizacionales, es un proceso que ha llevado a las entidades a repensar continuamente la forma en que diseñan su estructura organizacional y/o sus sistemas de trabajo. El éxito relativo en el desarrollo de sus emprendimientos sociales no parece haber estado condicionado en forma exclusiva por la estructura que sostiene su ejecución. Sin embargo, se presume que un sistema de trabajo poco flexible y altamente burocratizado o, por el contrario, con responsabilidades poco definidas, caótico e improvisado, podría haber hecho peligrar los más acertados emprendimientos por fallas en su ejecución (BID y SEKN, 2006).

Un factor general de todos los emprendimientos sociales entrevistados en este estudio, es que los cambios e innovaciones que se realizan están directamente relacionados con la adaptación al medio. En el caso de la Corporación Genera, "La gestión de cambio que la organización ha desarrollado está vinculada con el aprendizaje obtenido en todos estos años, el que ha llevado a flexibilizar las estrategias y tácticas para cumplir de mejor manera los objetivos. Por otro lado la corporación sostiene haber modificado algunas metas y objetivos, señalando que en un comienzo se proponían lograr nuevas leyes y normativas a nivel nacional, sin embargo debido a varias decepciones y a comprender que dichos cambios no depende de la gestión organizacional han modificado el foco de incidencia, centrándose en poner temas en la agenda pública y motivar a la ciudadanía en Pro de determinados temas”.

Otro caso interesante es el de la Corporación Raíces, "la organización ha sufrido grandes cambios condicionados por el desfinanciamiento padecido por las ONG a comienzo de la década del 90, con ello las Organi- 
zaciones No Gubernamentales de Chile dejaron de ser prioridad para el apoyo internacional, pasando gran parte de estas organizaciones a ser el brazo ejecutor del Estado o a desaparecer con los años. En lo que respecta a Raíces, la corporación ha subsistido cambiando sus objetivos y giros a lo largo de los últimos 15 años, de esta forma el antiguo colectivo encargado que abordaba las temáticas de genero, hoy en día lucha contra la explotación sexual de lo niños. Esto es uno de los principales factores que nos ayuda a sostener que Raíces posee una gran capacidad de cambio y adaptación, pudiendo en primero lugar subsistir al desfinanciamiento, para posteriormente encontrar un nuevo nicho de acción sustentable. Este cambio no ha estado ajeno a conflictos, de las 6 personas fundadoras que salieron de la Vicaria, hoy sólo quedan 3. Evidentemente la cotidianidad de muchas de las ONG es vivir en base a la inestabilidad presupuestaria, recibiendo grandes sumas en algunos proyectos para posteriormente tener extensos periodos de carencias. Sin embargo el merito de la "gestión de cambio" de Raíces, no es únicamente subsistir y salir a flote en momentos complejos, sino ser capaz de hacerlo con principios y convicciones. De esta manera la corporación no busca temáticas que están en la opinión publica y en la agenda gubernamental para obtener fuentes estables de financiamiento, sino que se relaciona con temas que nacen de investigaciones y estudios serios impulsados por la organización para posteriormente con los resultados, ellos buscar incidir en la opinión pública y buscar intervenir en la agenda gubernamental”.

Para el caso de las organizaciones comunitarias territoriales (Unión comunal de Juntas de Vecinos), estás han tenido múltiples falencias para adaptarse a la contingencia asociativa nacional, de esta manera, gran parte de estas organizaciones están compuestas por adultos mayores, y poseen dirigentes que ejercen poca participación y democracia. Sin embargo existen excepciones, como el caso de la Unión Comunal $\mathrm{N}^{\circ} 1$ de Maipú, quienes con la nueva directiva, "remediaron el conflicto, realizando cambios a través de una gestión colaborativa y participativa que permitiera motivar, adherir, ayudar, capacitar e incentivar a la participación para revertir el escenario adverso existente en la organización”.

En el caso de las organizaciones comunitarias funcionales, la gestión del cambio no existe como un proceso establecido, el cambio está orientado a las relaciones y a la labor intangible de enriquecimiento personal y comunitario. Esto se vincula estrechamente con lo planteado por el BID y SEKN (2006), donde se expone que es frecuente en las OSC (Organizaciones de la Sociedad Civil) la ausencia de políticas de gestión de personal, asociada a una cultura que valora la informalidad en los contactos personales cotidianos, y las relaciones de trabajo basadas en la proximidad personal. Los patrones culturales caracterizados por una mayor fluidez en las relaciones interpersonales y el espíritu de ayuda mutua, presentes en estos tipos de emprendimientos, pueden representar una característica distintiva que potencia la capacidad de la organización de generar los resultados anhelados. 


\section{Vínculos, colaboración y financiamiento:}

En Chile al igual que en Argentina, el sector sin fines de lucro se encuentra en un contexto complejo que no permite el avance y la evolución exitosa de estas organizaciones, algunos de los factores expuestos por $\mathrm{Pa}$ ladino, Milberg y Sánchez (2006), son: la ausencia de políticas públicas, la falta de profesionalización de la administración pública, la ausencia de un marco legal y fiscal apropiado y la falta de políticas públicas en general. También están: la corrupción, la inestabilidad de las reglas y la existencia de marcos legales que imponen trabas. Esta situación enfrenta a las OSC con un complicado escenario ya que no sólo necesitan que se revierta la pasividad del Estado si no que, más complicado aún, necesitan que se abandonen las acciones contraproducentes, especialmente las que son generadoras de mayor pobreza.

El estudio dio a conocer que la cooperación por parte del Estado es deficitaria. Algunos ejemplos que respaldan esta afirmación son:

Corporación Genera, "la cooperación de parte del Estado ha sido deficitaria, estableciendo un marco normativo de financiamiento y de constitución que dificulta el actuar de las ONG”.

Fundación Trascender, “a nivel Estatal existen algunos vínculos y colaboraciones con la División de Organizaciones Sociales (DOS) o a través del Fondo Mixto del ministerio de Planificación. Sin embargo la organización posee una gran crítica al mecanismo estatal de financiamiento, el cual señalan es inestable, reducido y limitado a proyectos puntuales, sin contribuir al desarrollo efectivo de la organización”.

Corporación Raíces, "Por un lado uno de los actores centrales es el Estado que a través del SENAME, financia los diversos programas y proyectos de la corporación. Sin embargo la relación con este servicio público no está exenta de problemas. La organización es crítica tanto de las metodologías de intervención de menores, como de las formas de financiamiento de las licitaciones y fondos concursables. Por su parte la corporación ha tenido problemas puntuales con el Estado, debido a que una investigación que evidenciaba la explotación de las temporeras y un plan piloto financiado por el SENAME, no fueron publicados, este último pudo ser masificado años después por UNICEF, evidentemente con ciertas modificaciones”.

Algunos integrantes del sector no lucrativo (Citados en: Paladino, Milberg y Sánchez, 2006), intentan dar alguna explicación al poco o nulo apoyo financiero que da el Estado a estas organizaciones; Beatriz Pellizzari, de la Unisina afirma que, desde el sector estatal se evidencia un desconocimiento del sector social y una imposibilidad de articular por la dinámica política y por la burocratización. De igual forma Eduardo Balán, de El Culebrón Timbal y Fabián Ferraro de Defensores del Chaco, consideran que existe un choque de intereses entre el estado y la misión misma de sus 
movimientos sociales. Parte de la misión de estas entidades es intervenir en la vida pública y por esto chocan con las instituciones públicas. Esto genera un conflicto de intereses que hace que a veces no se reciba apoyo por parte del Estado.

Por otro lado, las relaciones que se han dado con las Universidades han sido calificadas por las organizaciones sin fines de lucro como variables. Algunos ejemplos que confirman lo anterior son:

Fundación Integra, "la fundación realiza gestión colaborativa con la Universidad San Sebastián, con quien tiene un convenio de práctica profesional para alumnos de carreras relacionadas con la educación”.

Corporación Genera, “el rol con las universidades es variable, por un lado se han creado vínculos estrechos con algunos centros de alumnos y federaciones, buscando estrechar lazos con los estudiantes; también se han realizado colaboraciones con los estamentos académicos y directivos de la Universidad Católica Silva Henríquez, con quienes se trabajó en la campaña de "promoción de los derechos laborales. Sin embargo reconocen que la relación con las casas de estudio no es tan estrecha como debiese ser ya que las Universidades deben autofinanciarse, lo que se traduce en una incursión en el campo de los estudios, campañas sociales y otras actividades en las que compiten directamente con las ONG. Esta competencia es desleal debido a la gran diferencia de redes, ingresos, cobertura e infraestructura que existe entre las ONG y las Universidades”.

Fundación Trascender, "la relación con las universidades es más cercana pero no carente de problemas. La fundación se vincula principalmente con los alumnos practicantes y tesistas, que son quienes desarrollan un trabajo teórico-académico de alto impacto para la organización. Sin embargo, el proceso de acercamiento ha sido desde la fundación hacia la universidad, sin haberse logrado generar un vínculo permanente y estrecho".

En los últimos años se ha planteado con fuerza en muchas universidades que su tarea no puede limitarse a impartir formación, y desarrollar investigación, realizando acciones puntuales de extensión hacia la sociedad. Se les demanda especialmente en el mundo del desarrollo, pero la tendencia es global y asume a fondo la idea de Responsabilidad Social. La formación de los futuros graduados debe hacerse desde esa perspectiva, privilegiando su compromiso de servicio al país y al mundo. La investigación debe priorizar agendas conectadas con los grandes problemas de la sociedad. La extensión debe transformarse en un trabajo activo permanente en la comunidad promoviendo, y formando parte de alianzas estratégicas para transformar y mejorar la realidad (Paladino, Milberg y Sánchez, 2006).

Las relaciones con el sector privado son calificadas como cercanas y tensas debido a su vinculación directa con el financiamiento. Algunos ejemplos que respaldan lo anterior son: 
Fundación Víctor Jara, "la fundación trabaja en la articulación de redes con otras organizaciones de distintos rubros, es decir, la colaboración entre organizaciones es un hecho, no por un tema de gestión, sino por la clara determinación participativa, aglutinadora, y colectiva de la fundación, donde colaboran tanto personas, como instituciones barriales, vecinales, organizaciones artísticas, que juntos le dan vida a la idea, de hacer comunidad y espacios libres de expresión social desde el mundo popular”.

Corporación Raíces, “la organización posee una buena relación con el empresariado chileno, quienes aportan un gran porcentaje del financiamiento. Particularmente la corporación recibe la colaboración de la Fundación San Carlos del Maipo, asociada a Sociedad Canal del Maipo y Eléctrica Puntilla”.

Es importante resaltar que el sector lucrativo ha generado un gran apoyo económico para las corporaciones, fundaciones, ONGs, etc. Este punto dista de lo planteado por Paladino, Milberg y Sánchez, (2006), pues ellos afirman que, a pesar del desarrollo de la responsabilidad social empresarial, desde el lado de los emprendedores sociales se ve a las empresas de manera muy crítica. La falta de apoyo de los empresarios locales y los condicionamientos de los donantes podrían ser interpretados como signo de incomunicación e incomprensión entre ambos sectores.

La relación entre las fundaciones, las empresas, las organizaciones sociales y el mundo público se basa en una estrategia que apunta a no perder las oportunidades de vincularse con los diferentes sectores. Algunas empresas privadas se vinculan a la comunidad de una manera innovadora y de alto impacto social son: BCI, Almagro Financial Group, Ultramar, Brotec, la Fundación San Carlos del Maipo, Sociedad Canal del Maipo y Eléctrica Puntilla.

Sin embargo para la Unión Comunal Juntas de Vecinos No 1 de Maipú asegura que, "sigue estando en deuda la realización de un vínculo más permanente, la colaboración se traduce en una unión momentánea y puntual destinada a actividades específicos, pero no a la cohesión social destinada a objetivos compartidos. Esto redunda en que el trabajo comunitario sigua estando disgregado, sin una estrategia conjunta”.

\section{Sentido de pertenencia:}

Para estas organizaciones sin ánimo de lucro, el sentido de pertenencia es un tema fundamental, puesto que los profesionales que se vinculan con estas organizaciones en calidad de trabajadores, lo hacen porque se encuentran $100 \%$ comprometidos con la causa de la entidad a la que pertenecen, lo que facilita de alguna forma la ejecución exitosa de los proyectos y las actividades, pues cuentan con una disposición horaria flexible (a diferencia de las entidades privadas), como también con una perspectiva de sociedad y compromiso social, además de una orientación política social definida. 
Las siguientes citas tomadas de las entrevistas realizadas para este estudio, sirven para ejemplificar lo dicho anteriormente:

Fundación Trascender, "La misma razón de ser de cada organización es la encargada de motivar de manera individual a cada uno de sus miembros...”.

Fundación Asciende, "el sentido de pertenencia es esencial en la labor de la organización, el cual se consigue entregando responsabilidad, objetivos y tareas a las personas, interviniendo directamente en la motivación de los participantes y la continuidad que estos pueden tener en la organización”.

Fundación Integra, "El factor que permite un mayor vínculo entre la organización y los trabajadores es la vocación por la educación y el bienestar de los niños, vínculo que se refuerza al a los niños en riesgo social, víctimas de violencia intrafamiliar, abusos, vulneración de derechos, que asisten a los jardines y salas cuna de Integra”.

De igual forma el estudio realizado por el BID y SEKN (2006) también obtuvo como hallazgo que, en gran parte de las organizaciones de la muestra estudiada, se observa que las áreas dedicadas al desarrollo de los programas o proyectos, como aquellos de apoyo administrativo o técnico, generan un sentido de pertenencia entre sus miembros, este hecho promueve sinergias positivas debido a la interacción, pero también puede propiciar la emergencia de dos subculturas internas que no siempre conviven en total armonía.

\section{Comunicación}

La comunicación es un tema al que los emprendedores sociales les asignan una enorme importancia. Probablemente, esto no se deba sólo al impacto de la comunicación sobre los resultados, sino también al que tiene sobre la misión de su organización. Tan alta estima de la comunicación puede tener su fundamento en la necesidad de los emprendedores sociales de difundir sus ideas y proyectos. Esto también se relaciona con el hecho que las OSC funcionan para la sociedad; es decir que el destinatario final de sus acciones es la sociedad, por eso son organizaciones abiertas que funcionan con una lógica de apertura y que necesitan comunicar. Porque contrario a lo que se da en el sector privado, están deseosos de difundir su causa y de que les copien la idea si eso juega a favor de la causa (Paladino, Milberg y Sánchez, 2006).

Para los emprendimientos sociales entrevistados para este estudio, la comunicación se realiza a nivel interno de la organización y a nivel externo;

Unión Comunal Junta de Vecinos No 2 Maipú, “La comunicación interna formal se realiza cada 15 días o una vez al mes, cuando se junta la 
directiva en pleno a planificar y organizar el trabajo. Por su parte, se comunican para fines específicos e inmediatos vía mail, además de verse con bastante frecuencia en la misma Unión Comunal”.

Fundación COANIL, "La comunicación interna ha sido un tema complejo debido a que actualmente son 1.200 que están a lo largo de todo Chile. A veces la información no llega a todos los lugares como debería ser. Es una labor que recae en los coordinadores de los equipos de trabajo, en ellos está la tarea de transmitir bien la información. Esta organización ha privilegiado el mantener a todos informados, contar las cosas buenas y también las cosas malas. Existe una carta que envían una vez al mes a todos los empleados, a los socios, a la comunidad y al que sea necesario donde cuentan las cosas que han hecho y que están haciendo. Sus balances financieros están publicados en la página web y no todas las fundaciones los tienen publicados, además están auditados”.

Canal Comunitario La Victoria, “A nivel externo, para comunicarse y estar en contacto con otras organizaciones, dentro del medio de los canales comunitarios u otras instituciones o miembros de la sociedad civil y del gobierno, Señal 3 La Victoria posee una página web en la que describen quiénes son y de qué se trata el proyecto comunitario. A la vez, poseen un Facebook en el que publican sus eventos, opiniones y actividades. En ambas plataformas, se encuentra el correo de contacto de la organización, el que es revisado de manera constante”.

Corporación Genera, "la organización se centra en realizar una buena comunicación externa ya que se conjuga con los objetivos de la institución. Por ello la corporación se comunica a través de una página Web dinámica y una base de datos con el correo electrónico de múltiples adherentes a quienes se les envía información sobre las campañas y las diversas actividades realizadas. Además la organización posee Facebook, Twitter y news letter (herramienta para generar debate)".

Lo anterior se puede complementar con lo expuesto por el BID y SEKN (2006) donde afirman que, con el fin de hacer más eficaces los sistemas de comunicación en las OSC, en lo que se refiere a mejorar la imagen de la organización externamente y compartir valores internamente, es relevante el uso de herramientas de gestión que propicien una amplia y clara divulgación de metas, objetivos y resultados. Esto ayuda a fortalecer la identidad organizacional y su imagen pública.

Para las organizaciones comunitarias el tema de la comunicación es de vital importancia, tanto así que el Centro Comunitario 7 de Octubre cuenta con una persona encargada del tema de la comunicación, "La organización posee un encargado de comunicación, quien se ocupa de canalizar las distintas informaciones hacia los alumnos y hacia los profesores. Por otro lado, la comunicación destinada a propaganda se realiza interviniendo en cada una de las casas adyacentes al Centro, al respecto los miembros de la organización señalan que prefieren hablar particularmente con cada per- 
sona invitándola e inscribiéndola. Esta labor de trabajar persona a persona también la realizan cuando ven que el nivel de asistentes a clases diminuye, en tal caso asisten y se comunican con cada uno de los que no fueron, buscando saber las causas y motivarlos a asistir. El proceso propagandístico también lo realizan con afiches, pegando grandes cantidades en diversas zonas de su entorno local. Con respecto a medios modernos como Facebook, teléfono e Internet, estos son utilizados sólo como apoyo a la difusión”.

De igual forma el estudio realizado por Paladino, Milberg y Sánchez (2006) asegura que son muchos los emprendedores sociales que tienen un área o un responsable específico de comunicación. En cuanto al tipo de medios de comunicación utilizados no hay ninguno que se destaque sobre los otros.

\section{Liderazgo y planificación:}

La gran mayoría de las organizaciones entrevistadas cuentan con una estructura organizacional muy similar, por lo general son organigramas verticales conformados en primera instancia por un presidente, seguido de una Junta Directiva, un Gerente General y un Equipo Ejecutivo subdividido por áreas o coordinaciones. Esto coincide con el estudio realizado por el BID y SEKN (2006), donde el 95\% de las OSC investigadas predominan claramente una estructura organizacional que agrupa en dos grandes áreas a sus “direcciones” o “coordinaciones”. La primera está referida al conjunto de programas o servicios centrales ofrecidos por la organización y la segunda contiene las áreas administrativas o de gestión que apoyan tanto a los otros órganos de línea como a la institución en su conjunto.

La planificación de estas organizaciones por lo general se realiza a través del directorio, es decir, es el directorio el encargado de determina los lineamientos generales por los cuales se rige la organización, por ejemplo en el caso de la Corporación Forja, "la Junta Directiva determina los lineamientos generales por los cuales se rige la organización, donde la Directora Ejecutiva y el equipo de sicólogos ejecutan estas líneas de trabajo, sin embargo, las decisiones importantes se toman en conjunto puesto que, finalmente son ellos los que conocen la labor desde una perspectiva cercana aterrizando la realidad de Forja según el contacto con los niños y su desenvolvimiento en los talleres. En el ámbito de la planificación, pretenden llegar a todas las comunas del gran Santiago, no obstante, han trabajado con más de 40 colegios, fundamentalmente los que pertenecen a redes educacionales, aún cuando quieren llegar a regiones, saben que deben ir primero paso a paso".

Un caso similar es el de la Fundación Paternitas donde, “el Directorio es el ente encargado de fijar los lineamientos, también existen los jefes de área que son las personas encargadas de la coordinación de las diferentes actividades (áreas). Existen reuniones por cada área donde se conversa las actividades y talleres que se van a realizar y los resultados que se esperan obtener luego de cada taller. También estas reuniones servían para 
informar a todas las personas del equipo sobre los lineamientos de la organización”.

La Corporación Genera, “la corporación trabaja con Marco Lógico, tanto para cumplir con las exigencias internacionales de las instituciones cooperadoras, como para realizar un seguimiento de las actividades y acciones realizadas a través de indicadores de gestión”.

Lo anterior encuentra respaldo en el estudio del BID y SEKN (2006), donde aseguran que, para lograr un buen desempeño, estas organizaciones se valen de mecanismos de coordinación, tales como la conformación de comités de trabajo, la realización de reuniones periódicas donde exista intercambio de información, los procesos de supervisión y de seguimiento, etc. Estructuras organizativas de tipo matricial facilitan la conformación de equipos de trabajo por programas o por proyectos. Dos grandes áreas (coordinación y áreas de administración) se encuentran en el mismo nivel jerárquico y se realiza un trabajo descentralizado. Asimismo, el estudio de los casos de las OSC indica que resulta esencial promover el trabajo cercano entre las áreas técnicas que atienden los proyectos sociales y las áreas administrativas, de modo que ambas complementen sus esfuerzos y sus competencias en un proceso de aprendizaje conjunto y de esa manera comprendan el rol complementario que cada una juega en el proceso de generación de valor.

En el caso de las organizaciones comunitarias y juntas de vecinos los casos son muy similares, por ejemplo el Centro Comunitario 7 de Octubre, "La planificación se realiza a través de reuniones, en donde se deciden las actividades que se desarrollarán, generando la discusión para llegar a decisiones consensuadas que represente los deseos de cada uno de los miembros. Además la organización se encuentra dividida en tres áreas, lo que se traduce en tres personas encargadas de realizar y coordinar las actividades correspondientes a su sector. Sin embargo esta división por área, no exime la participación y responsabilidad de los integrantes en cada uno de los sectores, ni tampoco la toma de decisiones en forma grupal y consensuada”.

La planificación estratégica es importante en la gestión de las organizaciones, pues refleja la presencia de una visión a largo plazo, pero que una organización se rija por una planificación estratégica, no debe cerrarla a la posibilidad de asumir, en ciertos momentos, una estrategia emergente. Una relación dinámica y abierta con el entorno es fundamental para la definición del foco estratégico. Por lo general se peca más por falta de planificación que por falta de flexibilidad (BID y SEKN, 2006).

\section{Liderazgo y toma de decisiones}

El liderazgo que se da al interior de estas organizaciones presenta diversas perspectivas: una perspectiva externa donde las organizaciones se tienen que coordinar con los usuarios o clientes del servicio que prestan, al 
igual que con los voluntarios; y una perspectiva interna, donde las personas vinculadas laboralmente desarrollan un liderazgo colectivo, cada profesional posee la dirección de un área determinada, y esto permite la existencia de un diálogo entre pares.

Fundación Integra, "El liderazgo está dividido en dos planos, por un lado en el ámbito institucional está asociado a la investidura de un cargo, es decir la directora como cabeza de la institución ejerce el liderazgo del equipo. A nivel de trabajadores, los sindicatos existentes presentan líderes, los cuales representan y defienden los intereses de sus asociados"

Fundación Trascender, "El liderazgo al interior de la fundación posee diversas perspectivas. Por un lado en una perspectiva externa, la fundación al trabajar con organizaciones sociales se coordina y vincula constantemente con diversos liderazgos relacionados con las instituciones vinculadas. Por otro lado se relaciona con voluntarios, los que poseen un liderazgo reducido, esto se debe en gran medida a la ausencia de participación en la toma de decisiones organizacionales. Finalmente al interior de la organización, las 11 personas vinculadas laboralmente desarrollan un liderazgo colectivo, esto se debe a que cada profesional posee la jefatura de un área determinada, lo que permite un dialogo entre pares e iguales. De esta forma se puede apreciar que en las perspectivas interna (funcionarios) y externa (voluntarios), la organización presenta dos liderazgos totalmente distintos, siendo democrático en el primer nivel y autócrata en la segunda perspectiva”

Taller de Acción Comunitaria Valparaíso, “en el TAC la participación es de carácter formal, ya que se establecen jornadas de voluntarios, donde se discuten y evalúan las labores realizadas y por realizar, más las decisiones catalogadas como importantes son tomadas por un directorio, en donde participan representantes de organizaciones e instituciones, y directivos, en algunos casos también voluntarios. El directorio toma decisiones sobre materias de relevancia en el TAC, mientras que los voluntarios toman decisiones sobre las acciones correspondientes a los trabajos a realizar, como las planificaciones”.

En general las organizaciones estudiadas consideran que poseen un liderazgo democrático, el que está basado en el diálogo y en los equipos de trabajo que canalizan las percepciones y opiniones presentes en las diversas áreas.

Preuniversitario Rafael Maroto, "Es una participación directa donde cada persona interviene y da sus ideas y se desarrollan estas por toda la comunidad”.

Corporación Raíces, "La participación que se efectúa al interior de la organización es directa y altamente desarrollada, de esta manera como hemos señalado con anterioridad, los profesionales y trabajadores poseen la facultad de generar proyectos, innovar y realizar propuestas, las que se discuten colectivamente llegando a consensos. Sin embargo esto no queda 
sólo en la capacidad de propuesta o resolución de temas macro organizacionales, también las decisiones de gestión se realizan colectivamente”.

Lo anterior se complementa con lo expuesto por el BID y SEKN (2006), donde aseguran que no hacer la transición de un liderazgo directivo a uno participativo puede limitar el crecimiento y la eficiencia en la operación de los emprendimientos sociales. Los líderes deben ser consientes de que llegará el momento en que será necesario entregar las riendas a otros para que las iniciativas sociales florezcan. Esta conciencia puede ser complementada con el surgimiento de liderazgos laterales o equipos de trabajo que presionen al líder hacia la descentralización. Esto se relaciona directamente con la habilidad de delegar, que puede definirse como la capacidad de los líderes centrales para asignar a otros una serie de funciones, convirtiéndose en sus mentores y atribuyéndoles poderes para que asuman la responsabilidad de la operación de las unidades autónomas. Es necesario que el equipo humano que vaya a ejercer el liderazgo de estas organizaciones sea seleccionado cuidadosamente y tenga el potencial para convertirse en los posibles sucesores del líder central.

\section{Conclusiones}

\section{Gestión y recursos humanos}

El sector sin fines de lucro ha demostrado la necesidad de contar con personal profesional para la realización de sus actividades. Las temáticas que abordan estas organizaciones, exige contar con personas profesionales que sepan como proceder en las distintas problemáticas que sufren los sectores vulnerables chilenos, donde no hay cabida para la improvisación. El voluntariado sigue jugando un rol importante pero con responsabilidades menores, esto gracias a que posee un alto grado de movilidad, lo que dificulta la continuidad en los procesos.

Las organizaciones entrevistadas en este estudio, consideran como objetivo estratégico la gestión del conocimiento, sin embargo no existe ningún tipo de planificación sobre el tema. De ahí la importancia de contratar personal calificado y con ideas innovadoras. Por otro lado las universidades no han tomado en cuenta a estas organizaciones como potencial público objetivo, a esto se suma que el poco recurso con que cuentan las organizaciones son fines de lucro y las pocas o nulas alianzas de colaboración entre estas y las universidades, han dificultado mucho el tema de las capacitaciones al interior de las organizaciones.

\section{Adaptación al medio e innovación}

En general las organizaciones estudiadas han tenido que adaptarse a los distintos contextos. Esta claro que para poder tener una rápida adaptación en el medio es importante contar con una estructura interna flexible y 
descentralizada que permita operar con mayor agilidad y capacidad de respuesta. De todas maneras, al hablar de descentralizar las funciones de ejecución, es necesario que exista una coordinación entre la dirección y las diferentes áreas para que no exista un desempeño heterogéneo.

\section{Vínculos, colaboración y financiamiento}

Existe poca colaboración por parte del Estado hacia el sector no lucrativo, dichas organizaciones consideran que son un actor importante en las problemáticas sociales chilenas (salud, educación, inclusión social, etc), que cumplen un rol que debería estar en manos del Estado, pero que por su complejidad se le han escapado de las manos; y es la misma sociedad civil la que ha tenido que intervenir para llenar los vacíos que ha dejado el Estado. Esta misma razón es la que ha provocado que los emprendimientos sociales que participaron este estudio, sientan que a nivel económico el Estado este en deuda con ellos.

Respecto a las universidades, este estudio pretende dejar planteado un gran desafío para las entidades académicas. Invitándolas a conocer, familiarizarse y ser un actor activo en el complejo contexto que rodea al sector sin fines de lucro. Por otro lado el sector privado ha dado a conocer un cambio en su forma de pensar y en su manera de actuar, que se traduce en lo que se conoce como "responsabilidad social empresarial". Sin embargo, todavía es considerado como un aspecto que puede evolucionar mucho más, pero que de todas maneras, con el compromiso de algunos empresarios, se ha logrado sacar adelante a muchas organizaciones del sector.

Por último es importante resaltar que las organizaciones entrevistadas para este estudio, no están dispuestas a perder su independencia y autonomía por la consecución de su financiamiento.

\section{Sentido de pertenencia}

El sentido de pertenencia es el factor que moviliza el que hacer de estos emprendimientos. Como el recurso económico con el que se trabaja es escaso, esto demuestra que el trabajo realizado por cada individuo de la organización es un trabajo hecho con compromiso. Al mismo tiempo se hacen visibles en estas organizaciones la presencia de valores como la solidaridad, la ética, la confianza y la movilización para la acción. Las personas comparten los ideales de la organización y, por ello realizan el trabajo con compromiso, calidad y eficiencia.

\section{Comunicación}

La comunicación es una herramienta que permite brindar a todos los interesados e integrantes de los emprendimientos sociales, la imagen y confiabilidad, que toda actividad se desarrolla en pos de objetivos comunes con total despojo de subjetividades e individualidades. También cumple la función de demostrar que existe coherencia entre su manejo interno y 
las aspiraciones enunciadas en sus objetivos, coherencia entre el discurso y los comportamientos consecuentes. Además es un canal eficaz para desplegar esta coherencia con las motivaciones comunes de sus miembros, asegurando de esta forma fidelidad al mandato institucional.

\section{Planificación}

Los emprendimientos sociales entrevistados para este estudio, en su gran mayoría afirman cumplir con un procedimiento de planificación. Lo perciben como una herramienta necesaria para saber dónde es que se deben enfocar los esfuerzos y el trabajo. Sin embargo, no todos complementan esta planificación con un sistema de autoevaluación, reconocen la importancia de evaluarse constantemente, pero califican este proceso como un factor que se encuentra en una etapa básica, pero que día a día se nutre para poder evolucionar.

\section{Liderazgo y toma de decisiones}

La bibliografía utilizada en este artículo, expone que no existe un sólo tipo de liderazgo para la gestión de los emprendimientos sociales. En otras palabras no existe una receta mágica que indique cómo se debe gestionar la organización por parte del líder. Sin embargo aseguran que, dependiendo de la etapa de crecimiento en que se encuentren las organizaciones sin fines de lucro, el líder debe asumir diferentes roles.

Al inicio es necesario que el líder sea la cabeza y guía de la organización, encargado de la gestión, información, capacitación, consecución de los recursos, etc. Pero a medida que los emprendimientos sociales van evolucionando, se hace necesario que el líder descentralice su labor y la comparta con los coordinadores de cada área y con los jefes de proyecto. Esto genera estructuras flexibles al interior de las organizaciones, permitiendo una rápida respuesta a los diferentes requerimientos del entorno.

Por último es importante resaltar que el sector no lucrativo ha sufrido grandes cambios, ha dejado de ser ese lugar donde los grandes empresarios tiraban los pesos que les sobraban y donde sólo había cabida para un personal voluntario. Hoy día son compañías inteligentes, que logran transformar realidades en los escenarios más vulnerables de Chile, al mismo tiempo que generan un aprendizaje continuo al interior de la organización, un conocimiento que difícilmente podrían aprender en otro lugar que no fuera dentro de una organización sin fines de lucro. 


\section{Nota}

${ }^{1}$ Este artículo es parte del proyecto de investigación "Emprendimientos sociales y liderazgo en el sector no lucrativo chileno. Modelo de gestión y buenas prácticas.” Financiado por el Departamento de Investigación Científica y Tecnológica, DICYT, de la Universidad de Santiago de Chile. 


\section{Bibliografía}

Aedo, C. (1997), Organización industrial de la prestación de los servicios sociales, Ed. ILADES, Georgetown University, Estados Unidos.

Banco Interamericano de Desarrollo, Social Enterprice Knowledge network (2006), Gestión efectiva de emprendimientos sociales. Lecciones extraídas de empresas y organizaciones de la sociedad civil en Ibero América, Ed. Planeta, Estados Unidos.

Barea, J., Pulido, A. (2001), "El sector de instituciones sin fines de lucro en España”, CIRIEC - España, Revista de economía pública, social y cooperativa, $\mathrm{n}^{\circ} 37$, pp $35-49$.

Barrionuevo, L. (2001), “Empresas del tercer sector: bases para la instalación de un sistema de gestión”, en Cruzando fronteras: Tendencias de contabilidad directiva para el Siglo XXI. Del 4 al 6 de Julio de 2001. León, España. Universidad Católica de Argentina.

Comité Económico y Social Europeo (2007), La economía social en la Unión Europea, CIRIEC - Internacional, Bruselas.

De La Maza, G. (2003), “Sociedad Civil y Democracia”, Documento de Trabajo preparado para el Proyecto Regional Comparativo Sociedad Civil y Gobernabilidad Democrática en los Andes y Cono Sur de la Fundación Ford y el Departamento de Ciencias Sociales de la Pontificia Universidad Católica del Perú.

Drucker, P. (2001), Dirección de instituciones sin Fines de Lucro, Ed. El Ateneo, Argentina.

FUNDIBES (2009) Anuario Iberoamericano de la Economía Social. Ed. Fundación Iberoamericana de la Economía Social, Madrid.

Kliksberg, B. (2011), Emprendedores Sociales. Los que hacen la diferencia, Fundación Claritas, Ed. Temas, Argentina.

Maglieri, G. (1997), “Organizaciones sin fines de lucro”, FACES N 5, pp $95-127$.

Monzón, J. L. (2004) “Cooperativas, Economía Social y Globalización” en Economía Social y Cooperación al desarrollo en Iberoamérica. Papel de la Fundación Iberoamericana de la Economía Social, FUNDIBES, Madrid.

Paladino, M., Milberg, A., Sánchez, F. (2006), Emprendedores sociales y empresarios responsables. Ed. Temas, Buenos Aires Argentina.

PNUD (2004), El Poder: ¿para qué y para quién? Programa de Naciones 
Unidas para el Desarrollo, Santiago de Chile, Informe de Desarrollo Humano en Chile 2004.

PNUD-FOCUS (2006): Estudio Comparativo del Sector sin Fines de Lucro, John Hopkins Univeristy. Programa de Naciones Unidas para el Desarrollo, Santiago de Chile.

Radrigán, M., Barría, C. (2005), “Situación y Proyecciones de la Economía Social en Chile”, en Pérez de Uralde, J.M. Panorama de la Economía Social en Iberoamérica, editado por la Fundación Iberoamericana de la Economía Social. Fundación Iberoamericana de la Economía Social, FUNDIBES, Madrid

Vernis, A., Iglesias, M., Sanz, B., Solernos, M., Urgell, J., Vidal, P. (2004) La gestión de las organizaciones no lucrativa, Ed. DEUSTO, España. 